\title{
Interactive comment on "Best practices for precipitation sample storage for offline studies of ice nucleation" by Charlotte M. Beall et al.
}

Charlotte M. Beall et al.

cbeall@ucsd.edu

Received and published: 7 September 2020

The Authors wish to thank Referee 4 for their comments and discussion. We summarize our responses and changes made to the manuscript below. Note that line numbers refer to the latest, marked-up version of the manuscript:

Referee: "(1) Figure 1 and line 136: I am wondering, why the error bars shown are symmetrical. In a log-plot, I would assume unsymmetrical bars for symmetrical errors."

I am assuming that the referee is referring to the error bars on the most visible points (e.g. the points at -4 and $-4.75^{\circ} \mathrm{C}$ ). The error bars represent $95 \%$ binomial sampling intervals as in (Agresti and Coull, 1998), and are not symmetrical. I realize the figure is a bit congested and it isn't possible to see the individual data points. I considered 
decreasing the x-axis, but given the updates to Fig. 1, I think it is important to keep the full composite INP spectrum visible.

Referee: “(2) L.138: Section 3.2: I am missing storage experiments with 'pure' water, since we know from our own experiments that even deionized/distilled water can become ice nucleating after several days."

This is very interesting, but we have not experienced this ourselves, in DI water that we have stored for weeks in polypropylene bottles, and are not aware of any literature reporting such an effect in deionized, distilled or otherwise 'pure' water. For those reasons we did not carry out such a test.

Referee: “(3) L.192-193, L262-263 (conclusion), L.292-293: Apparently, there are significant deviations in the stored:fresh ratios, both above and below 1 . How can then simple correction factors be applied? In addition, I am highly skeptical about these correction factors: given that the actual correction factors are usually small (mostly between 0.9 and 1.8), they are likely much smaller than most other errors in such type of ice nucleation studies, and so their usefulness is questionable in my view. In addition, it is highly questionable whether these correction factors can be applied to studies at other locations, using different sampling and investigation methods, and studying different(marine) samples. I would very much prefer to see instead the uncorrected raw data then in such studies. In summary, I do not concur with conclusion no. 2. The authors also seem to be skeptical as they state in lines 292-293: "However, it remains to be seen how INP sensitivity to storage varies by environment or INP composition."'

Considering these issues and suggestions made by the other reviewers, we agree that the results are better represented as indications of the uncertainties associated with storage. Updates have been made to the tables, table legends and text to reflect this change:

Abstract: Finally, the estimated uncertainties associated with the 4 storage protocols are provided for untreated, heat-treated and filtered samples for INPs between -9 and

Interactive comment 
$-17^{\circ} \mathrm{C}$.

Conclusion, Line 375: "2. Estimates of uncertainty attributed to storage impacts and AMTD $95 \%$ confidence intervals for INP measurements obtained from stored samples are provided (see Tables 5-7)."

Legend Table 5: Table 5. Estimate of uncertainty associated with storage impacts for INPs with activation temperatures between -9 and $-17^{\circ} \mathrm{C}$ measured in stored, untreated precipitation samples. Confidence intervals were derived from the log-normal distribution of changes observed in INP concentrations due to storage (see Fig. 2 and details in Sect. 3.2). Temperature intervals where datapoints were too few to derive confidence intervals are indicated with "NA". Changes in INP concentration corresponding to enhancements or losses greater than 1 order of magnitude (losses $<=$ $-90 \%$ or enhancements $>=+900 \%$ ) in bold.

Legend Table 6: Table 6. Estimate of uncertainty associated with storage impacts for INPs with activation temperatures between -9 and $-17^{\circ} \mathrm{C}$ measured in stored, heattreated precipitation samples. Confidence intervals were derived from the log-normal distribution of changes observed in INP concentrations due to storage (see Fig. 3 and details in Sect. 3.2). Changes in INP concentration corresponding to enhancements or losses greater than 1 order of magnitude (losses $<=-90 \%$ or enhancements $>=$ $+900 \%$ ) in bold.

Legend Table 7: Table 7. Estimate of uncertainty associated with storage impacts for INPs with activation temperatures between -11 and $-19{ }^{\circ} \mathrm{C}$ measured in stored, filtered precipitation samples. Confidence intervals were derived from the log-normal distribution of changes observed in INP concentrations due to storage (see Fig. 2 and details in Sect. 3.2). Temperature intervals where datapoints were too few to derive confidence intervals are indicated with "NA". Changes in INP concentration corresponding to enhancements or losses greater than 1 order of magnitude (losses $<=-90 \%$ or enhancements $>=+900 \%$ ) in bold.

Interactive comment
Printer-friendly version

Discussion paper 
Referee: "Figure S1-S4: I do not understand what is plotted in Figures S1 through S4 in the supplement, and I am in doubt that it is correct. The captions say "INP losses or enhancements (\%) : : :" What are losses in \%? Shouldn't they be given as negative numbers? How can losses and enhancements be fitted simultaneously as a basis for correlation analysis, as the figure captions imply? Even if not losses in percent are meant but if loss factors are presented, then losses would imply values smaller than 1. However, in none of the figures S1-S4 is there any point below the 10ËẼ line. How can that be, as figures 2-4 of the main paper clearly show that losses do occur? Moreover, I am wondering whether plotting the losses or enhancements in percent does make sense at all. I think factors would be more suitable, because some of the changes are several orders of magnitudes. In particular for losses (not such much for enhancements), plotting them in percent may be misleading: for example losses by a factor of $10 \mathrm{E} E-2$ or $10 \mathrm{EE}_{-}-4$ (i.e., a difference of two orders of magnitude) would lead to a loss of nearly $-100 \%$ in both cases $(-99 \%$ or $-99.99 \%)$. Note that losses cannot be lower than $-100 \%$ !"

Thank you for bringing this to our attention. Figures S1-S4 were originally intended to show whether absolute change in INP concentration relates to the storage time interval, so that we could determine whether the magnitude of the change correlated in time, independent of the sign of the change. However, the referee brings up a good point that there is a problem of scale as losses approach (-) 100\%, but enhancements have no upper bound.

Figures S1-S4 have been updated as suggested using the INP change factors, and the text has been updated as follows (to reflect the updated correlation factors):

Abstract, Line 23: Correlations between total storage time (1-166 days) and changes in INP concentrations were weak across sampling protocols, with the exception of INPs with freezing temperatures $>=-9{ }^{\circ} \mathrm{C}$ in samples stored at room temperature. Sec. 3.2, Line 209: For INPs with freezing temperatures $>=-9{ }^{\circ} \mathrm{C}$ in samples stored at room temperature, time is moderately correlated with changes in INP concentrations $(R 2=$

Interactive comment 
Conclusions, Line 379: 4 . With the exception of warm-freezing INPs (freezing temperAMTD

atures $>=-9^{\circ} \mathrm{C}$ ) in samples stored at room temperature, we found little to no correlation between changes in INP concentrations and storage intervals on timescales between 1-166 days, indicating that most enhancements or losses are likely happening during freezing or on timescales $<24$ hours.

Interactive comment

Referee: "(5) L.88: "At the MESOM Laboratory parking lot: : :" To which of the two collection points given (lines 81-83) does this location belong?"

Corrected so that the lines referred to are consistent in how they refer to the 2nd location. Sec. 2.1, Line 113: At the Isaacs Hall location, an ISO 6706 plastic graduated cylinder and plastic funnel, $27 \mathrm{~cm}$ in diameter, was used for precipitation collection.

Referee: "(6) L.262-263: ": : : it is worth noting that freezing is lethal for most cells" This statement is too general. Note that INTRACELLULAR freezing is lethal for most cells, while EXTRACELLULAR freezing is often not critical and, thus, survived by freezetolerating species."

These lines have been removed due to this issue, and others, also brought to our attention by the other referees.

Referee: "(7) L.458 (caption to Fig.3): "measured in heated precipitation samples" When were the samples heated? Directly after collection, or just before measurement?"

Treatments were applied just prior to measurement. The following changes have been made to the text:

Sec. 2.2: Heat treatments and filters were applied to samples just prior to processing (i.e. treatments were not applied to samples prior to storage).

Printer-friendly version

Figure 3 Legend: Figure 3: Ratio of INP concentrations measured in heated precipita- 
tion samples (stored:fresh), calculated in successive $2{ }^{\circ} \mathrm{C}$ increments between -19 and $-7^{\circ} \mathrm{C}$. Same samples as shown in Figure 2, but heated to $95^{\circ} \mathrm{C}$ for 20 minutes just AMTD prior to measurement to eliminate heat-labile INPs (see Methods Sect. 2.2 for details).

Referee: "(8) L.468 (caption to Fig.4): "measured in filtered $(0.45 \mu \mathrm{m})$ precipitation samples" When were the samples filtered? Directly after collection, or just before measurement?"

This legend has also been updated:

Figure 4 Legend: Figure 4: Ratio of INP concentrations measured in filtered $(0.45 \mu \mathrm{m})$ precipitation samples (stored:fresh), calculated in successive $2^{\circ} \mathrm{C}$ increments between -19 and $-7^{\circ} \mathrm{C}$. Same samples as in Fig. 2 but filtered with a $0.45 \mu \mathrm{m}$ syringe filter prior to measurement (see Methods Sect. 2.2 for details).

Referee: "(9) Tables 5-7: Please provide a few sentences of explanation on the 95\% confidence interval limits. What exactly do these values imply and, more importantly, how can they be applied? For example, considering line 2 in Table 5: the suggested correction factor is 1.72. The confidence limits of this correction factor are 0.25 and 11.27, implying that the correction factor could also be significantly below 1 . I was wondering then, given this large confidence interval, whether it is useful at all to make such a correction (see also my comment 3 above)"

Tables 5-7 and text have been updated to reflect changes suggested in the referee's comment \#3. Additionally, the following has been added to the text to explain how these values may be interpreted:

Discussion, Line 289: While mean INP changes are within a factor of $\sim 2$ or less of fresh sample INP concentrations for all protocols except "Room temperature" (Table 5), none of the 4 storage protocols prevented significant losses or enhancements of INP concentrations in all samples (Fig. 2), indicating that INP concentration measurements on fresh precipitation are superior to measurements on stored samples. $95 \%$ confi- 
dence intervals in Table 5 span losses $>1$ order of magnitude in all protocols across multiple temperature intervals. These uncertainties equal or exceed INP measurement uncertainties (1-2 orders of magnitude) at temperatures $>-20{ }^{\circ} \mathrm{C}$ due to discrepancies between instruments (DeMott et al., 2017). If correspondence within 1 order of magnitude (or $2-3^{\circ} \mathrm{C}$ ) is desired, uncertainties associated with storage should also be considered in studies using samples from coastal or marine environments. Thus, uncertainty distributions provided in Tables 5-7 can be used to evaluate observed INP concentrations and responses to treatments in the context of potential changes due to storage. However, the degree to which INP sensitivity to storage varies by INP source (e.g. with soil-derived INP populations) remains to be tested.

Interactive comment on Atmos. Meas. Tech. Discuss., doi:10.5194/amt-2020-183, 2020. 

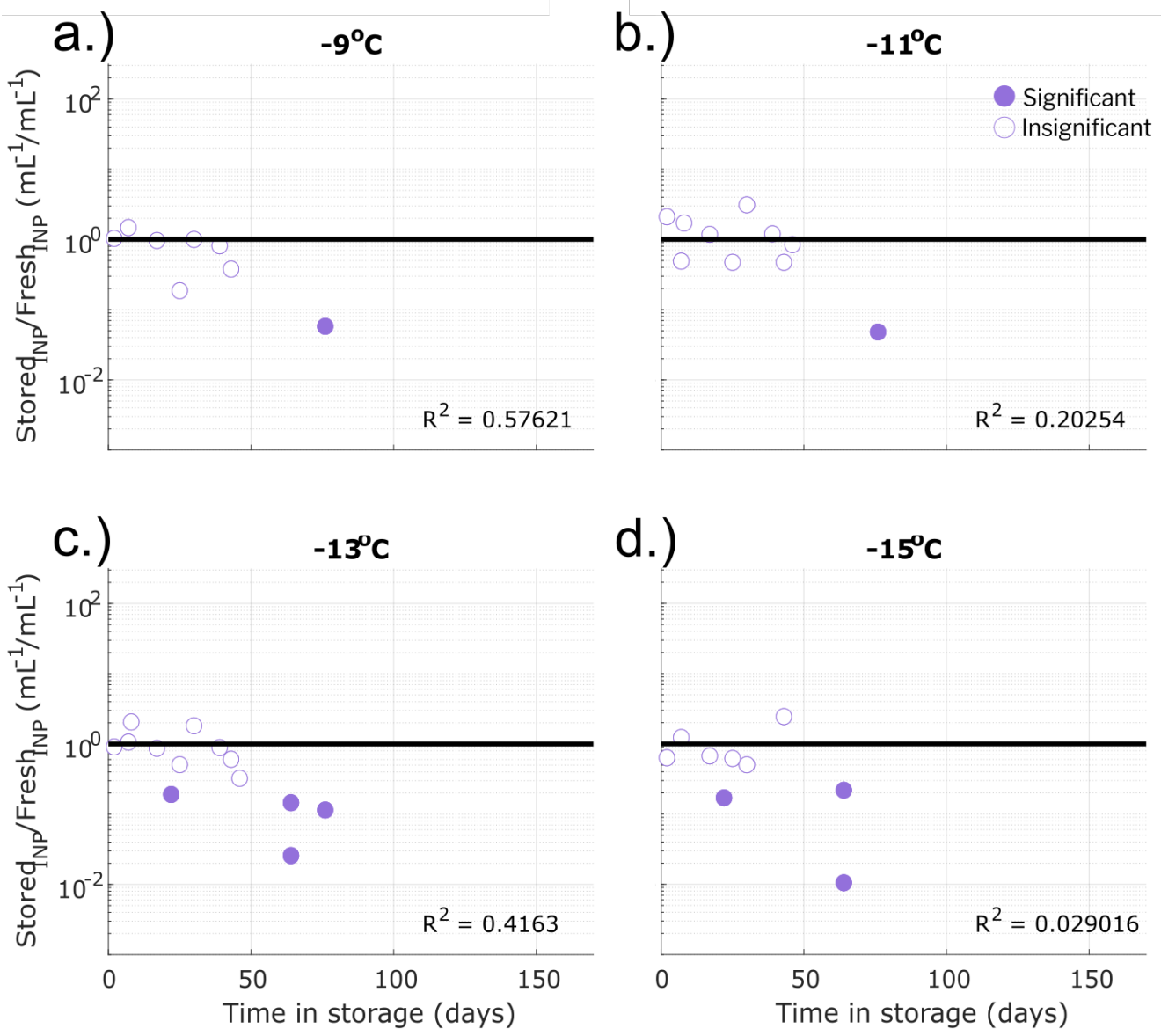

\section{Interactive comment}

Fig. 1. Figure $S 1$ 


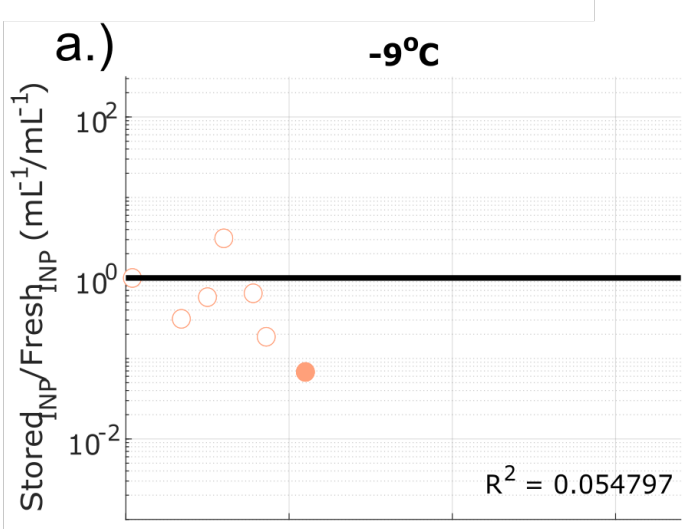

b.)

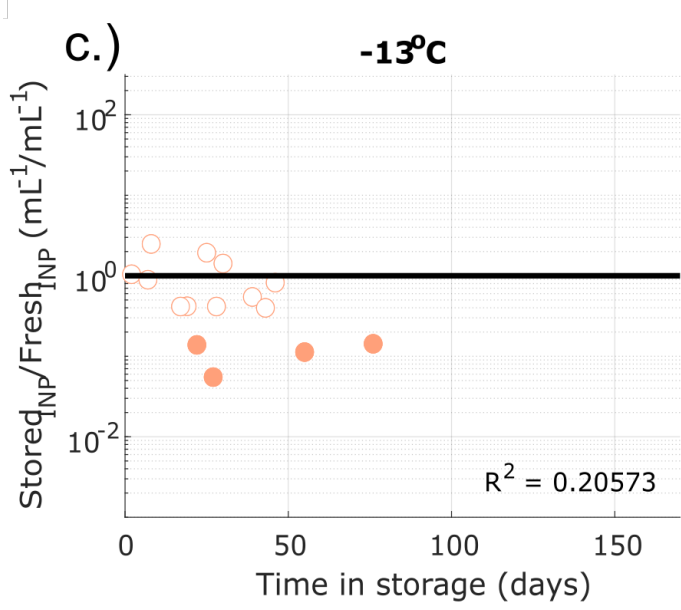

Fig. 2. Figure S2

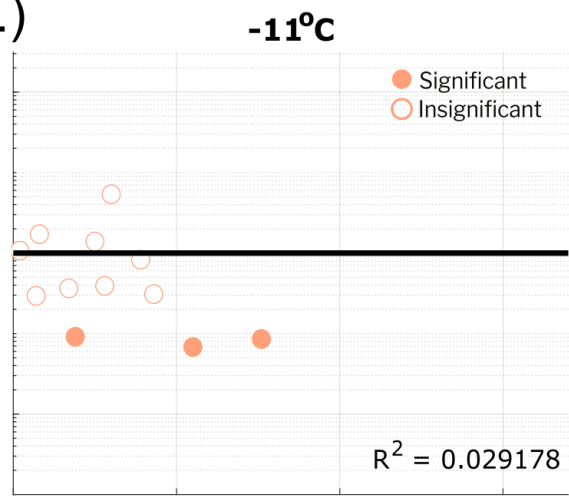

AMTD

Interactive comment

d.) $-15^{\circ} \mathrm{C}$

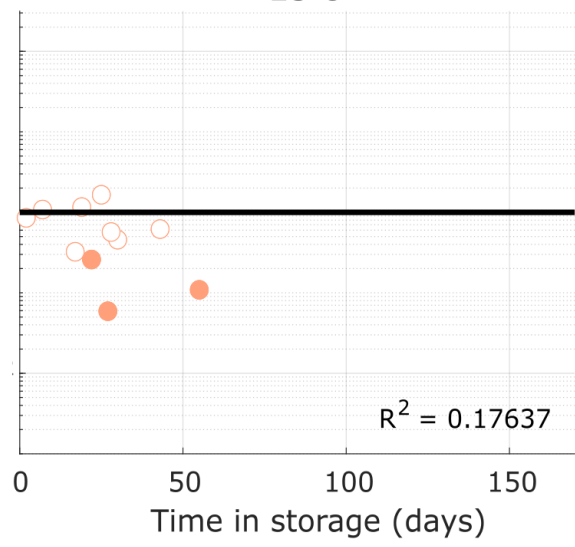

Printer-friendly version

Discussion paper

(c) (i) 
AMTD
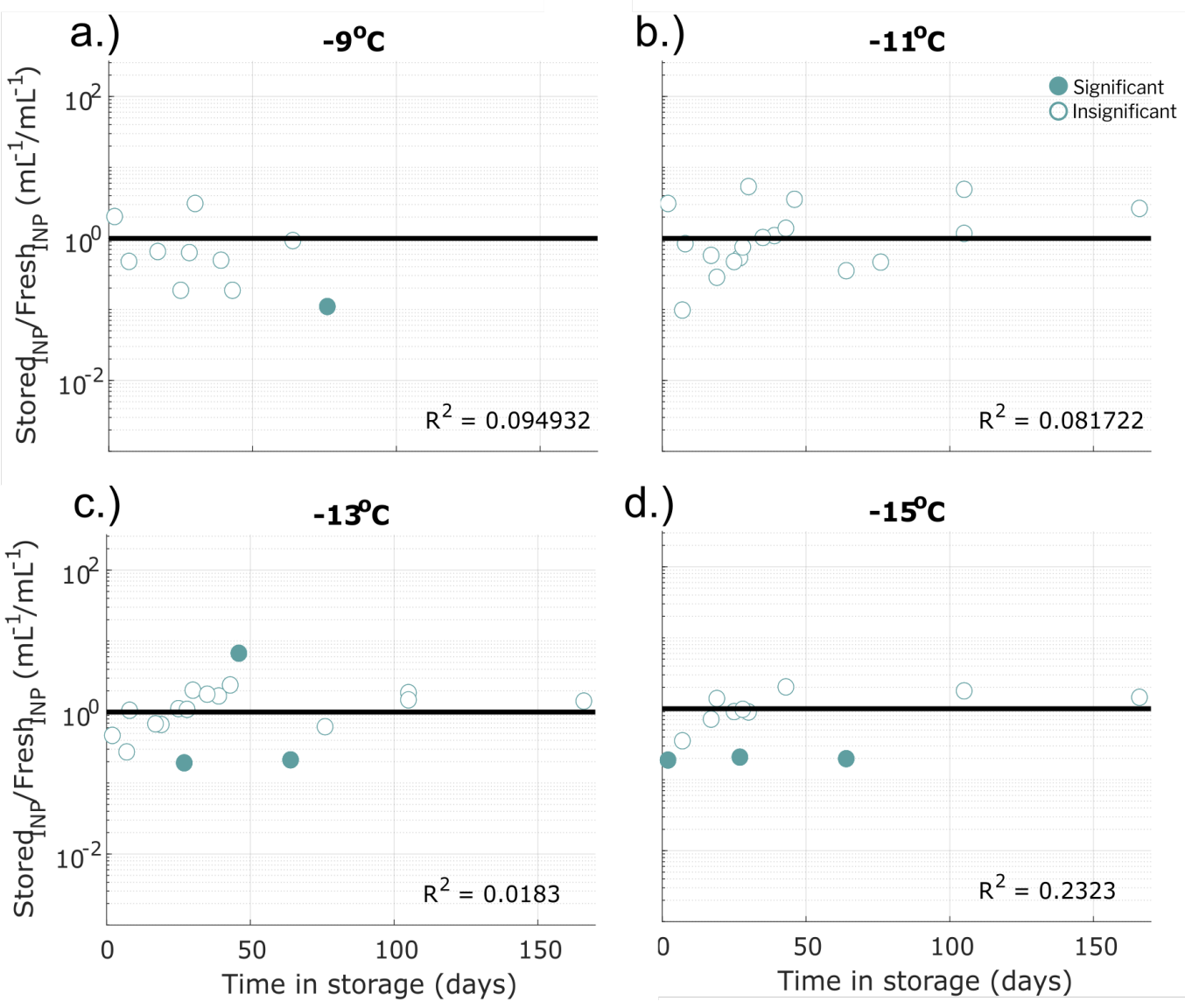

\section{Interactive comment}

Fig. 3. Figure $S 3$

Discussion paper 
AMTD

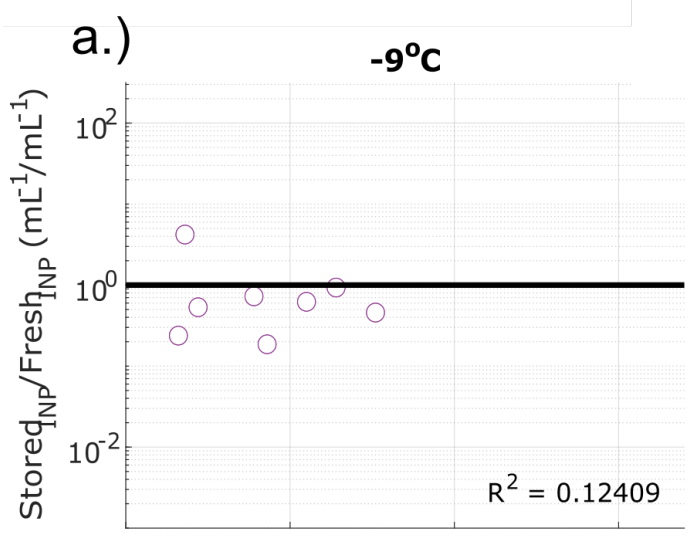

b.)
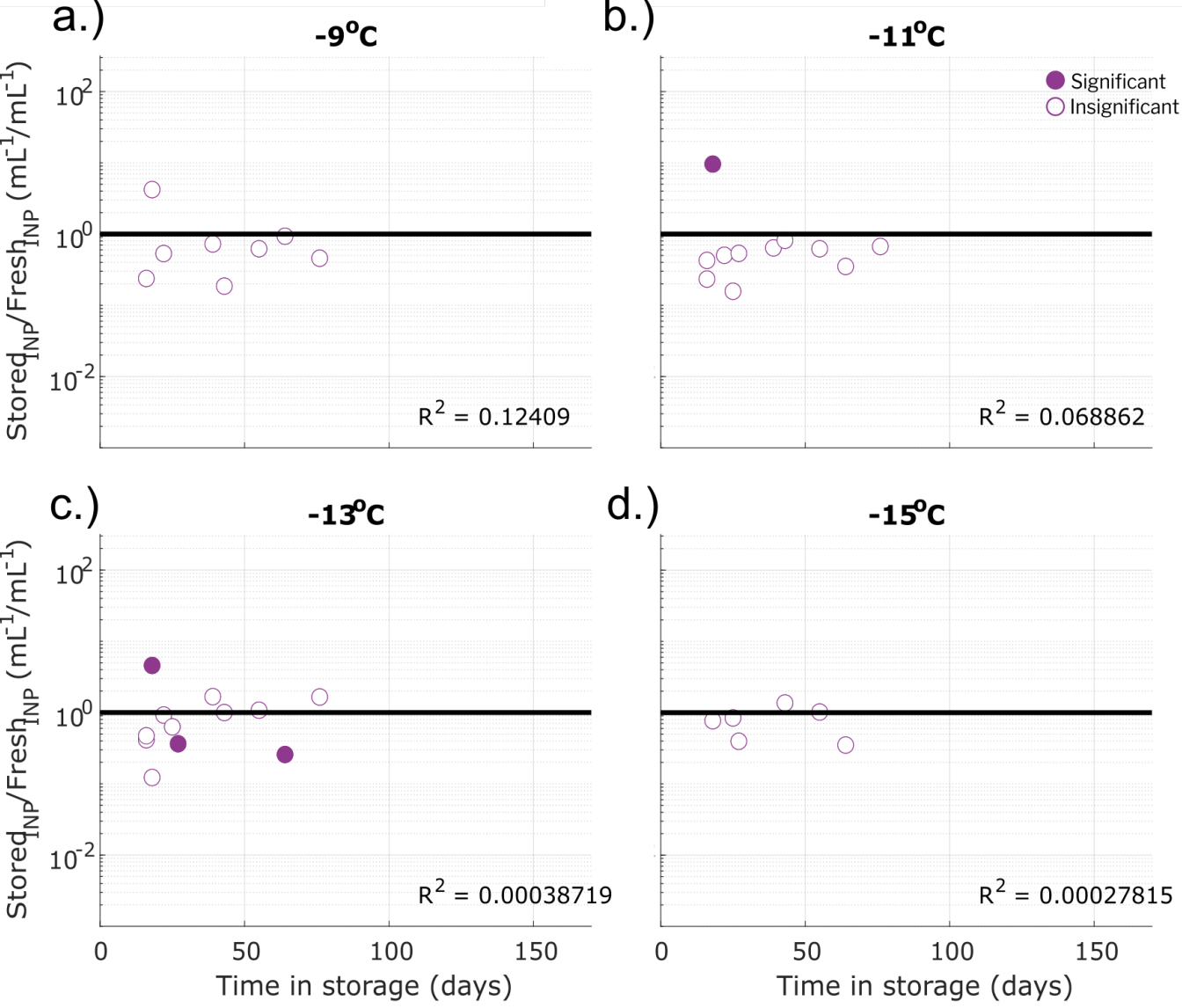

Fig. 4. Figure S4

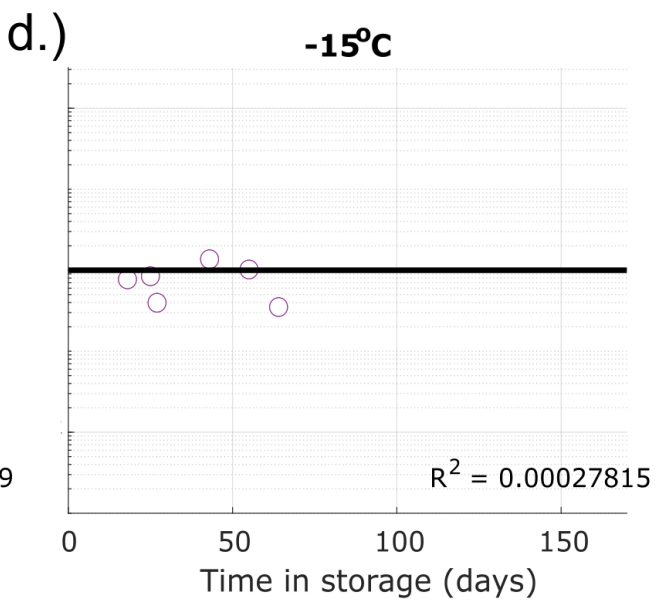

Printer-friendly version

Discussion paper

(c) (1) 